\title{
Seabed-Rock-Layer Electromagnetic Communication Channel Model with Low Path Loss Based on Evanescent Wave
}

\author{
Zhiqiang NIE ${ }^{1}$, Shilian $W A N G^{1}$, Dahai $C H E N^{2}$, Tian $D E N G^{1}$ \\ ${ }^{1}$ Dept. of Electronic Science, National University of Defense Technology, 109 Deya Road, 410073 Changsha, China \\ ${ }^{2}$ Inst. of Electronic Engineering, China Academy of Engineering Physics, 64 Mianshan Road, 621000 Mianyang, China \\ wangs1@nudt.edu.cn
}

Submitted August 25, 2017 / Accepted January 25, 2018

\begin{abstract}
The main limitation of the development of underwater wireless electromagnetic communication is severe attenuation introduced by the seawater characteristics of high permittivity and high conductivity. Fortunately, in previous studies, it was found through experiments that the loss between two underwater antennas near seawater surface or seabed is much smaller than the higher order severe attenuation for the line of sight (LOS) path in seawater. But no one has given reasonable explanation for this phenomenon. To solve this problem, we investigate the propagation mechanism of this phenomenon theoretically. The main component of seabed-rock-layer is basalt, an alternative seabed-rock-layer communication channel model based on evanescent wave generated by the total reflection on the seabed-rock-layer surface is proposed in this paper. Then we analyze the performance of this model according to Goos-Hanchen (GH) Shift of evanescent wave. Simulation results show that the path loss in this model is about 1/20 of that in seawater and the propagation velocity can be increased 20 times. Proposed technology is expected to become an important part of underwater high speed and reliable communication.
\end{abstract}

\section{Keywords}

Underwater electromagnetic communication, seabedrock-layer, evanescent wave, low loss, high speed

\section{Introduction}

In recent years, low loss, high reliability and high speed underwater wireless data transmission links are demanded urgently in civil and military domains such as petroleum exploration industry, marine environment monitoring and military command [1]. So the underwater wireless communication is an important topic in ocean that has attracted worldwide attention. Contemporary underwater acoustic communication, with long communication range of up to $20 \mathrm{~km}$, is widely used in most long-range under- water wireless data transmission applications [2]. However, narrow-band acoustic waves yield poor performance because of the susceptibility to multipath and Doppler effects, resulting in a significant limitation of the allowable bandwidth [3]. Fortunately, these problems can be overcome by reconsidering the use of electromagnetic communication in short-range transmission links due to the high bandwidth and strong anti-noise ability. Another option for underwater is optical communication, which has the greatest advantage of extremely large capacity [4]. Unfortunately, optical waves are severely affected by the scattering of suspended particles and marine plankton, and only deliver good performance in very clear water. In comparison with optical communication, electromagnetic wave can be operated under non-line-of-sight (NLOS) conditions and is insensitive to water turbidity, salt concentration and pressure gradients. Thus, electromagnetic communication is an ideal choice for underwater wireless communication applications.

The development of underwater wireless electromagnetic communication is mainly limited by severe attenuation. At the beginning of the study, researchers used extremely low frequency to launch electromagnetic wave in order to overcome the attenuation [5]. But very high transmitting power and large antenna size are required. Later, the researchers demonstrated that antennas coated with insulating material or wrapped inside the insulation cylinder shell for electromagnetic wave emission, which greatly improve launching efficiency [6], [7]. A. A. Abdou proved it is feasible to utilize electromagnetic wave in an underwater communication system using loop antennas [8]. However, these methods cannot solve the core problem of exponential attenuation of electromagnetic wave in seawater. Reference [9] referred to the use of surface wave produced by the propagation of electromagnetic wave going from seawater into the air, along the air-water interface, and finally down to seawater, whose path loss is much smaller than the higher order severe attenuation path in seawater and, hence, the communication range can even reach several kilometers. A. Shaw et al. took experiments to verify the formulation and found that the attenuation of electro- 
magnetic wave will become smaller if the antennas are in shallow ocean zone and the electromagnetic wave can spread over $90 \mathrm{~m}$ [6]. But the article did not give reasonable theoretical analysis and explanation for the propagation mechanism. Since the permittivity and conductivity of seabed-rock-layer are much lower than those of seawater, electromagnetic wave could be guided through the seabedrock-layer with the similar effect of surface wave for the deeply submerged transceiver. We exploit the effect to establish a low loss channel model for high speed underwater communication through the seabed-rock-layer path with theoretical derivation and simulation verification in this paper.

\section{Propagation Characteristics in Sea- water}

Seawater has a high permittivity and high conductivity so that the propagation characteristics of electromagnetic wave in the seawater medium are greatly different from in air. In this section, the attenuation constant, phase constant, propagation velocity and wavelength of electromagnetic wave in seawater are analyzed and discussed.

\subsection{Attenuation Constant and Phase Constant}

The propagation constant of electromagnetic wave in seawater [10], [11] is expressed as:

$$
\gamma=\omega \sqrt{\mathrm{j} \omega \mu(\sigma+\mathrm{j} \omega \varepsilon)}=\alpha+\mathrm{j} \beta
$$

where $\omega=2 \pi f, f$ is the propagation frequency, and $\sigma$ is conductivity. $\varepsilon=\varepsilon_{0} \varepsilon_{\mathrm{r}}$ accounts for permittivity, and the permittivity in vacuum is $\varepsilon_{0}=1 / \sqrt{c^{2} \mu_{0}} \cdot c$ is light speed, $\varepsilon_{\mathrm{r}}$ is relative permittivity, $\mu=\mu_{0} \mu_{\mathrm{r}}$ is permeability and $\mu_{0}=$ $1.26 \times 10^{-4} \mathrm{H} / \mathrm{m}$ is permeability in vacuum, we assume the seawater and seabed-rock-layer are non-ferromagnetic medium so the relative permeability $\mu_{\mathrm{r}}=1$.

The propagation constant has a real attenuation constant $\alpha(\mathrm{Np} / \mathrm{m})$ and imaginary phase constant $\beta(\mathrm{rad} / \mathrm{m})$ respectively calculated as:

$$
\begin{aligned}
& \alpha=\sqrt{\frac{\omega^{2} \mu \varepsilon}{2}} \sqrt{\sqrt{1+\left(\frac{\sigma}{\omega \varepsilon}\right)^{2}}-1}, \\
& \beta=\sqrt{\frac{\omega^{2} \mu \varepsilon}{2}} \sqrt{\sqrt{1+\left(\frac{\sigma}{\omega \varepsilon}\right)^{2}}+1} .
\end{aligned}
$$

Seawater is a good conductive medium due to the conductivity is $\sigma_{1}=4 \mathrm{~S} / \mathrm{m}$ and the permittivity is $\varepsilon_{\mathrm{r}}=81 \varepsilon_{0}$, so $\alpha$ and $\beta$ can be simplified as:

$$
\alpha=\beta \approx \sqrt{\pi f \mu \sigma} .
$$

It can be seen that the attenuation constant and phase constant in seawater are not only related to the characteristic parameters of conductivity, permeability and permittiv- ity, but also closely related to the frequency and increase with the frequency. Therefore, the higher frequencies of electromagnetic wave are not suitable for long-range communication in seawater.

\subsection{Wavelength}

The wavelength of electromagnetic wave in seawater [10] is expressed as:

$$
\lambda=\frac{2 \pi}{\beta}=\frac{1}{f \cdot \sqrt{\frac{\mu \varepsilon}{2}\left(\sqrt{1+\left(\frac{\sigma}{\omega \varepsilon}\right)^{2}+1}\right)}} \approx \sqrt{\frac{4 \pi}{f \mu \sigma}} .
$$

We can observe that the wavelength of electromagnetic wave is related to the frequency, the higher the frequency, the shorter the wavelength. It is found that the wavelength of $100 \mathrm{kHz}$ frequency in seawater is only $5 \mathrm{~m}$, while in the air it will reach $3000 \mathrm{~m}$. Therefore, the size of the antenna in seawater can be much smaller than that in air.

\subsection{Propagation Velocity}

The propagation velocity of electromagnetic wave [10] is expressed as:

$$
v_{\mathrm{p}}=\frac{\omega}{\beta}=1 / \sqrt{\frac{\mu \varepsilon}{2}\left(\sqrt{1+\left(\frac{\sigma}{\omega \varepsilon}\right)^{2}}+1\right)} \approx \sqrt{\frac{4 \pi f}{\mu \sigma}} .
$$

As the conductivity in seawater is not 0 , the propagation velocity is a function of frequency. The propagation velocity of electromagnetic wave in seawater, though less than the speed of light, increases with higher frequency. When the frequency is more than $140 \mathrm{kHz}$, the propagation velocity of electromagnetic wave in seawater is about 400 times faster than that of acoustic waves. This result is very impressive which indicates that underwater electromagnetic wave communication has the advantages of high speed, real-time in data transmission.

\section{Proposed Channel Model}

Seawater has characteristics of high permittivity and high conductivity such that the propagation range of the electromagnetic wave through the seawater path is limited. In [12], a wavelength-compensated path loss model was proposed. It found that a low frequency electromagnetic wave with $1 \mathrm{kHz}$ would have an attenuation loss of $140 \mathrm{~dB}$ when spreading $50 \mathrm{~m}$ in seawater path. High absorption loss is the inherent characteristics of the seawater medium. But the electromagnetic waves which cross the seawaterair interface are very sharply refracted between two shallowly underwater antennas and the waves will be guided through a low loss path leading from one antenna directly up to the surface, along the surface, and directly down to 
the another antenna. Similarly, for the deeply submerged transceiver, there will be the same advantage for electromagnetic wave propagated through the interface between seawater and seabed-rock-layer [13]. Therefore, we can optimize the channel model by changing the propagation path for the deeply submerged transceiver. In this section we analyze the evanescent wave effect produced by the total reflection in seabed-rock-layer, and optimize the channel model for underwater wireless electromagnetic communication from five stages of propagation.

\subsection{Propagation Mechanism}

Antenna is an important factor for the transmission of underwater electromagnetic wave communication. Directly putting the any metallic antenna into the seawater for electromagnetic wave emission will cause short-circuit. Most of the researchers use circular antenna which coated with insulating material (such as PTFE layer) or wrapped inside the insulation cylinder shell for underwater experiments. The circular antenna will match the impedance of the seawater due to the permittivity of insulation materials is close to the seawater, which can greatly improve the emission efficiency [6], [7], [14], [15]. The main component of seabed-rock-layer is basalt, whose standard permittivity is $\varepsilon_{2}=8 \varepsilon_{0}$, conductivity is $\sigma_{2}=0.01 \mathrm{~S} / \mathrm{m}$ which are far less than the parameters of seawater. Long-range underwater communication cannot be realized through seawater path at present, however, when the transceiver is located near the deep-sea bottom, the electromagnetic wave can be propagated through the seabed-rock-layer due to the evanescent wave generated by the total reflection, and the path loss can be much smaller than that of the seawater path, which is deduced and analyzed theoretically in this section.

The propagation paths of electromagnetic wave between submerged antennas near deep-sea bottom are shown in Fig. 1. Electromagnetic wave which belongs to uniform planar electromagnetic wave emitted by the antenna is obliquely injected into the seabed-rock-layer through seawater. In any polarization, the wave can be decomposed

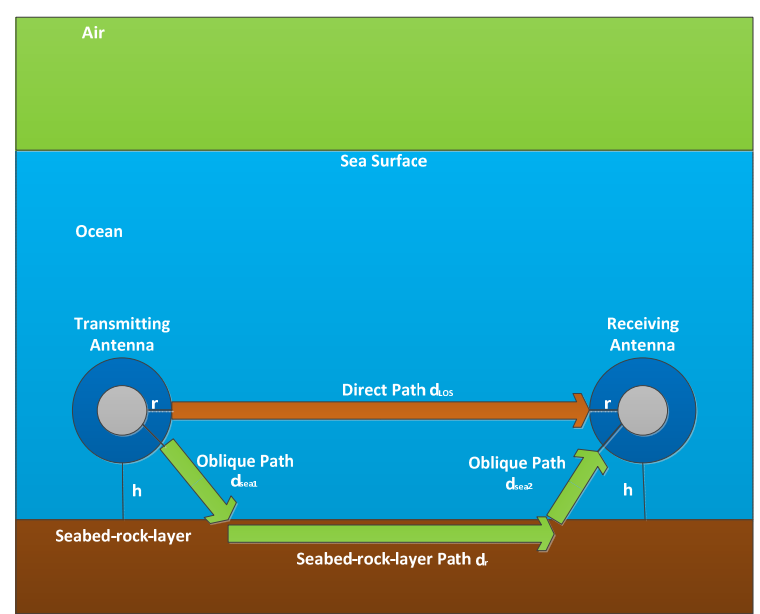

Fig. 1. Propagation paths between submerged antennas near deep-water bottom.

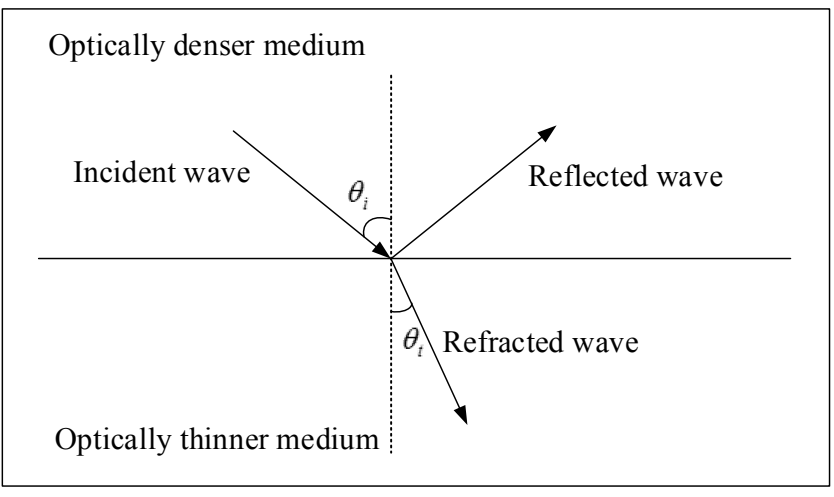

Fig. 2. Schematic diagram of Snell's law.

into two orthogonal linearly polarized waves [16]. The polarization direction of one is perpendicular to the incident plane known as vertical polarization wave $\boldsymbol{E}_{\mathrm{s}}$; another polarization direction in the incident plane known as parallel polarization wave $\boldsymbol{E}_{\mathrm{p}}$, that is: $\boldsymbol{E}=\boldsymbol{E}_{\mathrm{s}}+\boldsymbol{E}_{\mathrm{p}}$.

The schematic diagram of Snell's law is shown in Fig. 2. Snell' s law [15] is defined as:

$$
k_{\mathrm{lc}} \sin \theta_{\mathrm{i}}=k_{2 \mathrm{c}} \sin \theta_{\mathrm{t}}
$$

where $k_{i c}=w \sqrt{\mu \varepsilon_{i c}}, \theta_{\mathrm{i}}$ is incident angle, $\theta_{\mathrm{t}}$ is refraction angle, and $\varepsilon_{i \mathrm{c}}=\varepsilon_{i}-\mathrm{j} \sigma_{i} / \omega$ is complex permittivity, $i=1,2$ represents seawater and seabed-rock-layer, respectively.

The critical angle of total reflection is defined as $\theta_{\mathrm{c}}=\arcsin \sqrt{\varepsilon_{2 \mathrm{c}} / \varepsilon_{1 \mathrm{c}}}, \varepsilon_{i \mathrm{c}}$ is a complex number, so the critical angle is also a complex angle. When $f<<22.5 \mathrm{MHz}$, we obtain $\sigma_{2} / \omega \varepsilon_{2}>>1$, indicating that the seabed-rock-layer is also good conductive medium, and there is a real critical angle

$$
\theta_{\mathrm{c}}=\arcsin \sqrt{\frac{\varepsilon_{2}-\mathrm{j} \sigma_{2} / \omega}{\varepsilon_{1}-\mathrm{j} \sigma_{1} / \omega}}=\arcsin \left(\sqrt{\frac{\sigma_{2}}{\sigma_{1}}}\right)=2.866^{\circ}
$$

showing that a very small angle will cause the total reflection at the interface. When $\theta_{\mathrm{i}} \geq \theta_{\mathrm{c}}$

$$
\cos \theta_{\mathrm{t}}= \pm \sqrt{1-\sin ^{2} \theta_{\mathrm{t}}}= \pm \mathrm{j} \sqrt{\frac{\sigma_{1}}{\sigma_{2}} \sin ^{2} \theta_{\mathrm{i}}-1}
$$

Considering the electric field in the infinity tends to 0 , we take the negative sign. Then

$$
\cos \theta_{\mathrm{t}}=-\mathrm{j} \alpha^{*}
$$

where $\alpha^{*}=\sqrt{\sigma_{1} \sin ^{2} \theta_{i} / \sigma_{2}-1}$.

For the two kinds of non-ferromagnetic and conductive mediums, the transmission coefficient and the reflection coefficient of vertical polarized wave (TE wave) and parallel polarized wave (TM wave) are slightly different at the interface [16].

For TE wave, the transmission and the reflection coefficient are, respectively: 


$$
\begin{gathered}
\tau_{\mathrm{s}}=\frac{2 \cos \theta_{\mathrm{i}}}{\cos \theta_{\mathrm{i}}+\mathrm{j} \sqrt{\sin ^{2} \theta_{\mathrm{i}}-\frac{\sigma_{2}}{\sigma_{1}}}}, \\
\Gamma_{\mathrm{s}}=\frac{\cos \theta_{\mathrm{i}}-\mathrm{j} \sqrt{\sin ^{2} \theta_{\mathrm{i}}-\frac{\sigma_{2}}{\sigma_{1}}}}{\cos \theta_{\mathrm{i}}+\mathrm{j} \sqrt{\sin ^{2} \theta_{\mathrm{i}}-\frac{\sigma_{2}}{\sigma_{1}}}}=\left|\Gamma_{\mathrm{s}}\right| \exp \left(\mathrm{j} \varphi_{\mathrm{s}}\right) .
\end{gathered}
$$

Hence the amplitude and phase of the reflection coefficient are obtained, respectively

$$
\begin{gathered}
\left|\Gamma_{\mathrm{s}}\right|=1, \\
\varphi_{\mathrm{s}}=2 \arctan \left(\frac{\sqrt{\sin ^{2} \theta_{\mathrm{i}}-\frac{\sigma_{2}}{\sigma_{1}}}}{\cos \theta_{\mathrm{i}}}\right) .
\end{gathered}
$$

For TM wave, the transmission and the reflection coefficient are, respectively

$$
\begin{gathered}
\tau_{\mathrm{p}}=\frac{2 \sqrt{\frac{\sigma_{2}}{\sigma_{1}}} \cos \theta_{\mathrm{i}}}{\frac{\sigma_{2}}{\sigma_{1}} \cos \theta_{\mathrm{i}}+\mathrm{j} \sqrt{\sin ^{2} \theta_{\mathrm{i}}-\frac{\sigma_{2}}{\sigma_{1}}}}, \\
\Gamma_{\mathrm{p}}=\frac{\frac{\sigma_{2}}{\sigma_{1}} \cos \theta_{\mathrm{i}}-\mathrm{j} \sqrt{\sin ^{2} \theta_{\mathrm{i}}-\frac{\sigma_{2}}{\sigma_{1}}}}{\frac{\sigma_{2}}{\sigma_{1}} \cos \theta_{\mathrm{i}}+\mathrm{j} \sqrt{\sin ^{2} \theta_{\mathrm{i}}-\frac{\sigma_{2}}{\sigma_{1}}}}\left|\Gamma_{\mathrm{p}}\right| \mathrm{e}^{\mathrm{j} \varphi_{\mathrm{p}}} .
\end{gathered}
$$

Hence the amplitude and phase of the reflection coefficient are obtained, respectively:

$$
\begin{gathered}
\left|\Gamma_{\mathrm{s}}\right|=1, \\
\varphi_{\mathrm{p}}=2 \arctan \left(\frac{\sqrt{\sin ^{2} \theta_{\mathrm{i}}-\frac{\sigma_{2}}{\sigma_{1}}}}{\cos \theta_{\mathrm{i}} \cdot \frac{\sigma_{2}}{\sigma_{1}}}\right)
\end{gathered}
$$

where $\varphi_{j}\left(\theta_{j}\right)$ is the phase of the reflection coefficient, $j=\mathrm{s}, \mathrm{p}$ represents TE wave and TM wave, respectively. From the above equations of TE wave and TM wave we can see when the total reflection occurs between the two conductive mediums (seawater and seabed-rock-layer) interface, we get $\left|\tau_{j}\right| \neq 0$, It indicates that there is transmission wave in seabed-rock-layer that is different from the total reflection on the ideal conductor surface (no transmission wave). Furthermore, the phase of the reflected wave is changed. Under the precondition of total reflection, the electric field intensity of the transmitted wave in seabedrock-layer is:

$$
\boldsymbol{E}_{2}=\tau E_{\mathrm{i} 0} \exp \left[-\mathrm{j} \boldsymbol{k}_{\mathrm{t}} \times \boldsymbol{r}\right]=\tau E_{\mathrm{i} 0} \exp \left[-\mathrm{j} k_{2 \mathrm{c}} \boldsymbol{a}_{\boldsymbol{k} \boldsymbol{t}} \times \boldsymbol{r}\right]
$$

where $\boldsymbol{E}_{\mathrm{i} 0}$ is electric field intensity of incident wave, $\boldsymbol{a}_{\mathbf{k t}}=\boldsymbol{a}_{\mathrm{x}} \sin \theta_{\mathrm{t}}+\boldsymbol{a}_{\mathrm{z}} \cos \theta_{\mathrm{t}}$ is the unit propagation vector of plane electromagnetic wave and $\boldsymbol{r}=\boldsymbol{a}_{\mathrm{x}} x+\boldsymbol{a}_{\mathrm{z}} z$ is the vector of equal phase plane.

Combining (1) and (19), the electric field intensity of the transmission wave can be expressed as:

$$
\begin{aligned}
& \boldsymbol{E}_{2}= \\
& \tau E_{\mathrm{i} 0} \exp \left[-\left(\beta_{2}-\mathrm{j} \alpha_{2}\right) \alpha^{*} z\right] \cdot \exp \left[-\left(\alpha_{1}+\mathrm{j} \beta_{1}\right) \sin \theta_{\mathrm{i}} x\right]
\end{aligned}
$$

and the magnetic field intensity of transmission wave can be expressed as:

$$
\boldsymbol{H}_{2}=\frac{\boldsymbol{a}_{\mathrm{kt}}}{\eta_{2 \mathrm{c}}} \times \boldsymbol{E}_{2}
$$

where $\eta_{\text {ic }}=\sqrt{\mu_{\mathrm{i}} / \varepsilon_{\text {ic }}}=\left|\eta_{\mathrm{ic}}\right| \mathrm{e}^{\mathrm{j} \varphi_{\mathrm{i}}}$ is complex intrinsic impedance.

Therefore, the power flow density in seabed-rocklayer can be expressed as:

$\boldsymbol{S}_{\mathrm{av}, 2}=\frac{1}{2} \operatorname{Re}\left[\boldsymbol{E}_{2} \times \boldsymbol{H}_{2}^{*}\right]=$

$\boldsymbol{a}_{\mathrm{x}} \frac{\left(|\tau|\left|E_{\mathrm{i} 0}\right|\right)^{2}}{2\left|\eta_{2 \mathrm{c}}\right|} \cos \varphi_{2} \cdot \exp \left[-2\left(\beta_{2} \alpha^{*} z+\alpha_{1} \sin \theta_{\mathrm{i}} x\right)\right] \cdot \sqrt{\frac{\sigma_{1}}{\sigma_{2}}} \sin \theta_{\mathrm{t}}$

From (22) we can see that the power flow of the transmission wave goes along the tangential direction ( $\mathrm{x}$ axis) that is the seabed-rock-layer surface; the field amplitude attenuates exponentially along the normal z-direction (z-axis) whose effective depth generally has the same order of magnitude of skin depth [16]. Within the effective depth, the instantaneous power of transmission wave is not 0 , but in the extremely thick seabed-rock-layer the mean power along the $\mathrm{z}$-axis is 0 , which indicates that the exponential attenuation along the $\mathrm{z}$-axis is different from attenuation caused by ohmic-loss, there is no real power propagation. The transmission wave cannot penetrate into seabedrock-layer which merely belongs to the pure wave effect, and is named evanescent wave.

From (20) and (22), we can see that the propagation direction of evanescent wave is along the x-axis, and its attenuation constant, phase constant and propagation velocity are expressed as

$$
\begin{aligned}
& \alpha_{\mathrm{r}}=\alpha_{1} \sin \theta_{\mathrm{i}}, \\
& \beta_{\mathrm{r}}=\beta_{1} \sin \theta_{\mathrm{i}}, \\
& v_{\mathrm{p} 2}=\omega / \beta_{\mathrm{r}} .
\end{aligned}
$$

The propagation characteristics of electromagnetic wave in seabed-rock-layer are closely related to the incident angle $\theta_{1}$. As $\sin \theta_{1} \leq 1$, the path loss in seabed-rocklayer path is smaller and the propagation velocity is faster than that in seawater path.

When the deeply submerged transmitting and receiving antennas are extremely far apart away from each other, 
the signal in the LOS-seawater path will be severely attenuated and can be ignored, thus the propagation mechanism can be regarded as the following processes:

- Firstly, the electromagnetic waves travel through the insulating shell outside the antenna into seawater.

- Secondly, the waves which cross the interface between seawater and seabed-rock-layer are very sharply refracted and establish evanescent wave field in seabed-rock-layer.

- Thirdly, the waves will be guided along the seabedrock-layer surface with attenuation, and are completely absorbed within the effective depth.

- Fourthly, the absorbed energy stimulates the forced vibrations of the molecules or atoms in the medium, resulting in Rayleigh scattering.

- Fifthly, the Rayleigh waves generated by scattering are coherent, and finally the waves return to the seawater through the interface.

\subsection{Achievable Communication Range}

The lateral shift of evanescent wave on the seabedrock-layer surface is closely related to the earliest researcher F. Goos and H. Hanchen [17] who took experiment with multiple reflection method to prove when the total reflection occurs at the interface of two mediums, the actual reflection point has a lateral shift from the incident point, and later the lateral shift was named Goos-Hanchen (GH) Shift [18].

According to Static Phase Method [19], we can derive GH Shift expressed as:

$$
d_{\mathrm{r}-j}=-\frac{\lambda}{2 \pi} \frac{\partial \varphi_{j}\left(\theta_{\mathrm{i}}\right)}{\partial \theta_{\mathrm{i}}}
$$

where the subscript $\mathrm{r}-j=\mathrm{r}-\mathrm{s}, \mathrm{r}-\mathrm{p} . d_{\mathrm{r}-j}=d_{\mathrm{r}-\mathrm{s}}, d_{\mathrm{r}-\mathrm{p}}$ represent GH Shift of TE wave and TM wave, respectively.

Therefore the GH Shift is related to the polarization state of incident wave [20]. We can derive GH Shift of TE wave and TM wave, respectively:

$$
\begin{gathered}
d_{\mathrm{r}-\mathrm{s}}=\frac{\lambda_{1}}{\pi} \cdot \frac{\left(\frac{\sigma_{1}}{\sigma_{2}}-1\right) \sin \theta_{\mathrm{i}}}{\left(\Delta \sin ^{2} \theta_{\mathrm{i}}\right)^{1 / 2}\left[\cos ^{2} \theta_{\mathrm{i}}+\left(\frac{\sigma_{1}}{\sigma_{2}}\right)^{2}\left(\Delta \sin ^{2} \theta_{\mathrm{i}}\right)\right]}, \\
d_{\mathrm{r}-\mathrm{p}}=\frac{\lambda_{1}}{\pi} \cdot \frac{\sin \theta_{\mathrm{i}}}{\left(\Delta \sin ^{2} \theta_{\mathrm{i}}\right)^{1 / 2}}
\end{gathered}
$$

where $\Delta \sin ^{2} \theta_{\mathrm{i}}=\sin ^{2} \theta_{\mathrm{i}}-\sin ^{2} \theta_{\mathrm{c}}$.

Obviously, the GH Shift is determined by the incident angle $\theta_{1}$. If the incident angle is just equal to the critical angle $\theta_{c}$, the lateral GH Shift will reach infinity and the penetration depth will be infinite thus no more evanescent wave occurs. Only when the incident angle is larger than the critical angle, the lateral GH Shift exists, but the larger the incident angle is, the smaller the lateral GH Shift is. Therefore, when the incident angle is larger than and close to the critical angle, the lateral GH Shift of the wave at the seabedrock-layer surface can reach up to several kilometers. Theoretically this method can solve the problem of range-limitation in the seawater.

Through the above-mentioned derivation and analysis about the total reflection, we can conclude that the achievable horizontal communication range of the deeply submerged transceiver can be expressed as:

$$
d_{\mathrm{Los}}=d_{\mathrm{r}-\mathrm{j}}+2(h+r) \tan \theta_{\mathrm{i}}-2 r
$$

where $r$ is the radius of the insulating shell and $h$ is the vertical range of antennas from seabed-rock-layer.

\subsection{Path Loss}

Friis propagation equation can be expressed as [21]

$$
P_{\mathrm{r}}(\mathrm{dB})=P_{\mathrm{t}}(\mathrm{dB})+G_{\mathrm{t}}(\mathrm{dB})+G_{\mathrm{r}}(\mathrm{dB})-L_{\text {Loss }}(\mathrm{dB})
$$

where $P_{\mathrm{t}}$ and $P_{\mathrm{r}}$ are transmitted and received power, respectively. $G_{\mathrm{t}}$ and $G_{\mathrm{r}}$ are the antenna gains of the transmitter and receiver, respectively. And $L_{\text {Loss }}$ is path loss. For the seabed-rock-layer channel model, the path loss is related to absorption loss $L_{\text {att }}(\mathrm{dB})$ and spread loss $L_{\text {spread }}(\mathrm{dB})$ in two mediums.

$$
L_{\text {Loss }}(\mathrm{dB})=L_{\text {att }}(\mathrm{dB})+L_{\text {spread }}(\mathrm{dB}) .
$$

The absorption loss in the conductive mediums exists in three stages: seawater, seabed-rock-layer and returnseawater. The propagation range in seawater is set as $2 d_{\mathrm{s}}=2\left[(h+r) / \cos \theta_{1}-r\right]$ and the attenuation constant is $\alpha_{1}$. And in seabed-rock-layer, the propagation range is $\mathrm{GH}$ Shift $d_{\mathrm{r}}$ and the attenuation constant is $\alpha_{\mathrm{r}}$. Hence, the absorption loss can be calculated as:

$$
L_{\text {att }}(\mathrm{dB})=20 \log \mathrm{e}^{2 \alpha_{1} d_{\mathrm{s}}+\alpha_{\mathrm{r}} d_{\mathrm{r}}} .
$$

Most of the previous studies have focused on shortrange underwater wireless transmission scenarios and the spread loss is neglected. In this section, we deduce and analyze the characteristics of spread loss in long-range seabed-rock-layer channel model theoretically.

The insulating shell outside the antenna matches the impedance of the seawater, so the wavelength in both mediums is same. However, the permittivity and conductivity of seabed-rock-layer are different from those of seawater, so the wavelength will change due to the change of the phase constant caused by the propagation of evanescent wave on the seabed-rock-layer surface.

The path loss in vacuum is

$$
L_{0}(\mathrm{~dB})=20 \log \left(4 \pi d / \lambda_{0}\right)
$$




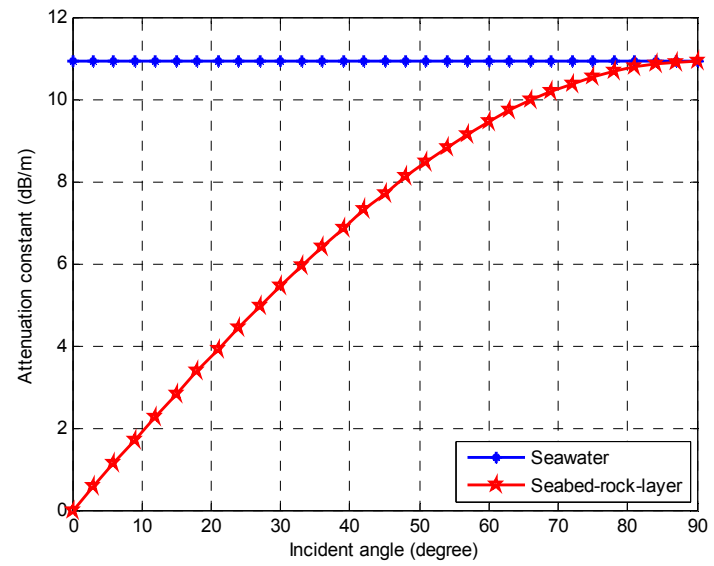

Fig. 3. Attenuation constant at frequency $100 \mathrm{kHz}$.

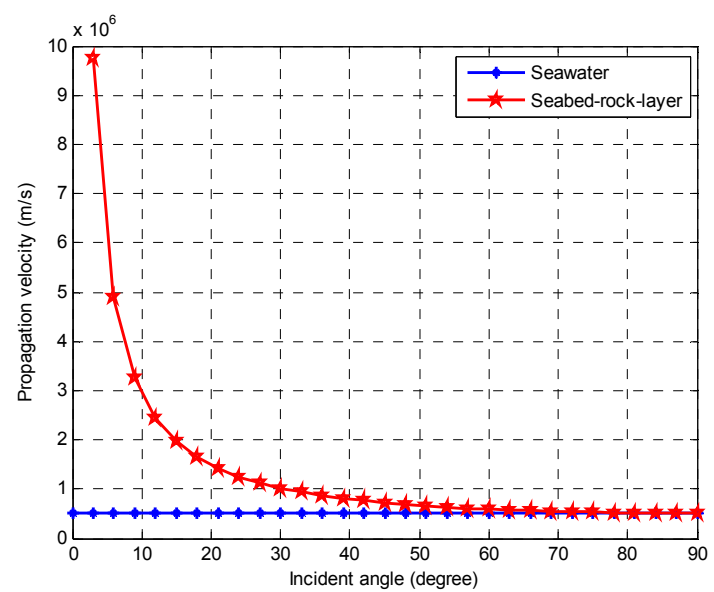

Fig. 4. Propagation velocity at frequency $100 \mathrm{kHz}$.

where $\lambda_{0}=c / f$ is wavelength in vacuum. The loss due to the change of the transmission medium [22] is

$$
L_{\mathrm{c}}(\mathrm{dB})=20 \log \left(\lambda_{0} / \lambda\right)
$$

where $\lambda$ is wavelength in conductive medium. Substituting (34) into (33) yields

$$
L_{\text {spread }}(\mathrm{dB})=L_{0}+L_{\mathrm{c}}=20 \log (4 \pi d / \lambda) .
$$

Combining (5) and (35), the spread loss of electromagnetic wave in conductive medium can be expressed as:

$$
L_{\text {spread }}(\mathrm{dB})=20 \log (2 \beta d) \text {. }
$$

Since the propagation of electromagnetic wave in the seabed model will go through five processes including insulating shell, seawater, seabed-rock-layer, return-seawater and insulating shell. Thus the spread loss can be corrected as:

$$
L_{\text {spread }}(\mathrm{dB})=20 \log \left[2 \beta_{1}\left(2 r+2 d_{\mathrm{s}}+d_{\mathrm{r}}\right)\right] .
$$

It is obvious that the influence factors of the path loss are not only the permittivity, conductivity, permeability of the conductive medium, but also the launch frequency, the incidence angle of electromagnetic wave, and the radius of the insulation cylinder shell as well as the vertical height of the antennas from the seabed-rock-layer surface.

\subsection{Channel Response}

Combining (19), (31), (32) and (37), we can get the received electric field intensity, expressed as:

$$
\begin{aligned}
E & =\frac{E_{0}}{2 \beta_{1}\left(2 r+2 d_{\mathrm{s}}+d_{\mathrm{r}}\right)} \mathrm{e}^{-\mathrm{j} 2 k_{1} 2 d_{\mathrm{s}}} \mathrm{e}^{-\mathrm{j} 2 k_{2} d_{\mathrm{r}}} \\
& =\frac{E_{0}}{2 \beta_{1}\left(2 r+2 d_{\mathrm{s}}+d_{\mathrm{r}}\right)} \mathrm{e}^{-2 \alpha_{1} 2 d_{\mathrm{s}}} \mathrm{e}^{-\mathrm{j} 2 \beta_{1} 2 d_{\mathrm{s}}} \mathrm{e}^{-2 \alpha_{2} d_{\mathrm{r}}} \mathrm{e}^{-\mathrm{j} 2 \beta_{2} d_{\mathrm{r}}} \\
& =\frac{E_{0}}{2 \beta_{1}\left(2 r+2 d_{\mathrm{s}}+d_{\mathrm{r}}\right)} \mathrm{e}^{-\alpha_{1}\left(2 d_{\mathrm{s}}+\sin \theta_{\mathrm{i}} d_{\mathrm{r}}\right)} \mathrm{e}^{-\mathrm{j} \beta_{1}\left(2 d_{\mathrm{s}}+\sin \theta_{\mathrm{i}} d_{\mathrm{r}}\right)} .
\end{aligned}
$$

Thus the amplitude-frequency response and phasefrequency response of the communication channel model can be obtained, respectively:

$$
\begin{gathered}
|H(f)|=\frac{1}{2 \beta_{1}\left(2 r+2 d_{\mathrm{s}}+d_{\mathrm{r}}\right)} \mathrm{e}^{-\alpha_{1}\left(2 d_{\mathrm{s}}+\sin \theta_{\mathrm{i}} d_{\mathrm{r}}\right)}, \\
\varphi(f)=-\beta_{1}\left(2 d_{\mathrm{s}}+\sin \theta_{\mathrm{i}} d_{\mathrm{r}}\right) .
\end{gathered}
$$

\section{Simulation Results and Analysis}

TE wave and TM wave are different only in GH Shift equations, but their characteristics are same. In this section, TE wave is utilized for simulation verification and analysis. The radius of the insulating shell is considered to be 9-16 mm [23]. For the frequency less than $1 \mathrm{GHz}$, the spread loss in the insulating shell is small enough to be ignored. In the deep sea, the vertical height of the antennas from the seabed-rock-layer surface is generally set to $0-3 \mathrm{~m}$, and we adopt the frequency of $100 \mathrm{kHz}$ primarily for simulation.

From Fig. 3, we can know that the attenuation constant in seawater is of $11 \mathrm{~dB} / \mathrm{m}$ or so. When the incident angle is greater than and close to the critical angle, it is about $0.5 \mathrm{~dB} / \mathrm{m}$ in seabed-rock-layer which is only $1 / 20$ of the attenuation in the seawater path. As can be seen from Fig. 4, the propagation velocity of electromagnetic wave in seawater is $0.5 \times 10^{6} \mathrm{~m} / \mathrm{s}$. However, with the same frequency and angle of incidence, the corresponding velocity in seabed-rock-layer can reach $9.8 \times 10^{6} \mathrm{~m} / \mathrm{s}$. It indicates that the communication range and propagation velocity will increase about 20 times through the seabed-rock-layer path.

In Fig. 5, the lateral GH Shift along the seabed-rocklayer surface is shown as a function of the incident angle. When the incident angle is greater than 3 degrees, the $\mathrm{GH}$ Shift is so small that there is no display. The isotropic antenna can emit the omni-directional waves, which will cause different GH Shifts when arriving at the interface between seawater and seabed-rock-layer. The larger the incident angle is, the smaller the lateral GH Shift is. When the incident angle is larger than and close to the critical angle, the lateral $\mathrm{GH}$ Shift can reach 300 meters at frequency $100 \mathrm{kHz}$ and more than two kilometers at frequency $1 \mathrm{kHz}$. 


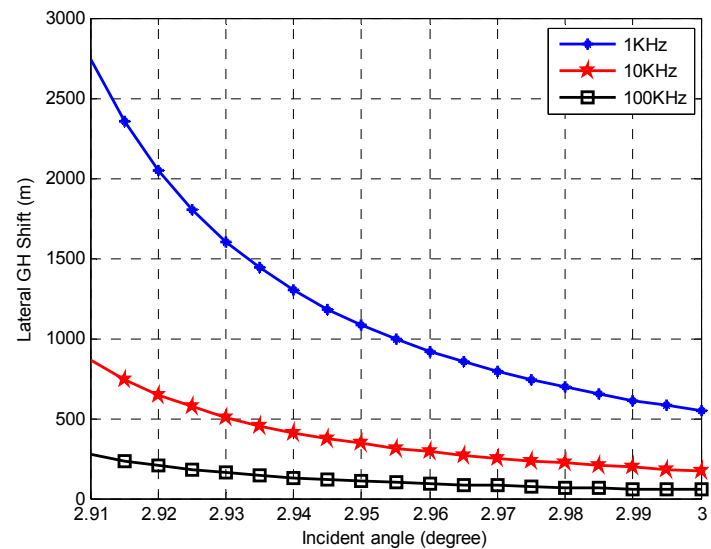

Fig. 5. Lateral GH Shift along the seabed-rock-layer surface at frequency $1 \mathrm{kHz}, 10 \mathrm{kHz}$ and $100 \mathrm{kHz}$.

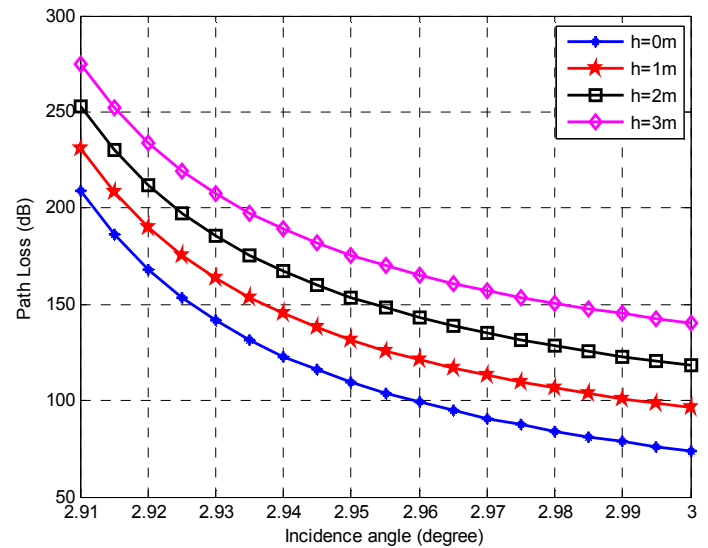

Fig. 6. Path loss in seabed-rock-layer communication channel model at frequency $100 \mathrm{KHz}$ with the different heights.

Figure 6 shows the characteristics of the path loss in the seabed-rock-layer communication channel model with different antenna heights. The higher the antenna, the greater is the path loss. As the height increases, the path loss increases by about $25 \mathrm{~dB}$ for each additional height of $1 \mathrm{~m}$. This is because the higher the height, the longer is the oblique range through the seawater before the electromagnetic wave arrives at the seabed-rock-layer, resulting in larger loss, especially the absorption loss. So much closer the antenna to the seabed-rock-layer, the more advantages of this channel model have.

Taking into account the case where the transmitting and receiving antennas are in contact with the seabed $(h=$ $0)$, the analysis of the characteristics of path loss is carried out. The results are shown in Fig. 7. It is found that the absorption loss of the conductive medium is smaller than the spread loss when the incident angle is greater than 2.97 degrees; the reason is that the GH Shift gets shorter when the incident angle is greater from Fig. 5. A slight change of the incident angle will cause a dramatic change in path loss. When the incident angle is about 2.92 degrees, the path loss is about $170 \mathrm{~dB}$.

According to Fig. 8 we can also see that if we adopt the lower frequency for communication, $1 \mathrm{kHz}$ for example,

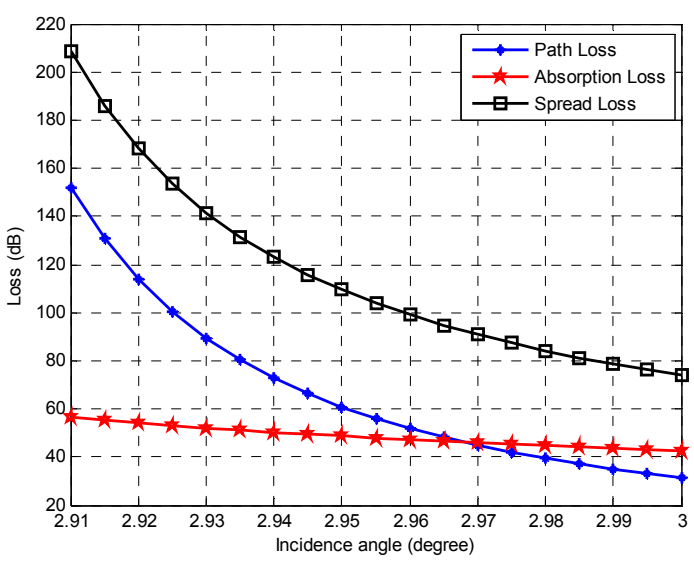

Fig. 7. The loss including path loss, absorption loss, spread loss in seabed-rock-layer communication channel model at frequency $100 \mathrm{kHz}$

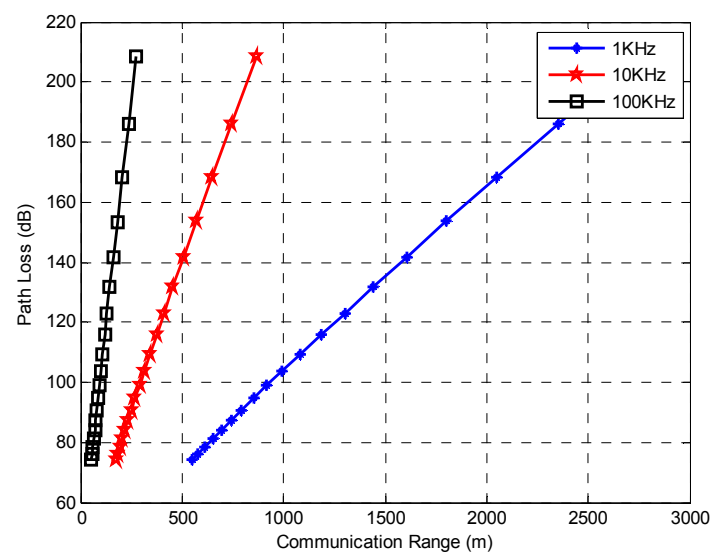

Fig. 8. Path loss in seabed-rock-layer communication channel model at frequency $1 \mathrm{kHz}, 10 \mathrm{kHz}$ and $100 \mathrm{kHz}$.

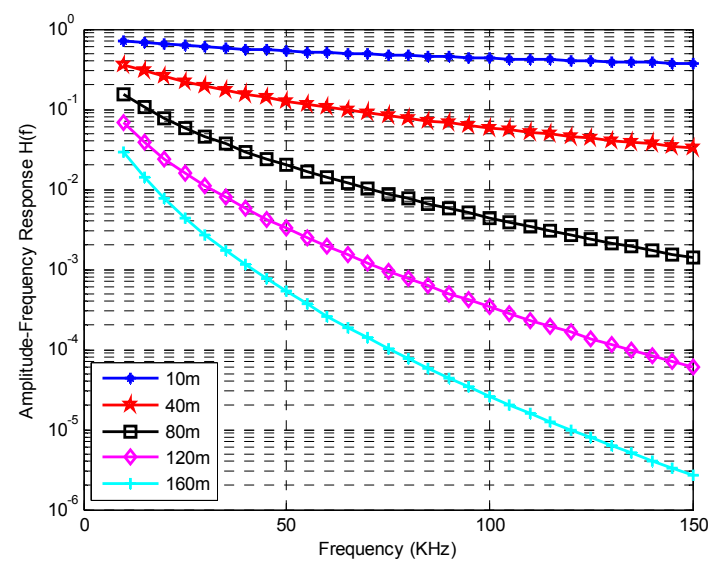

Fig. 9. Amplitude-frequency response.

the communication range can reach $2000 \mathrm{~m}$ farther, which is very important to break through the limitation of range when electromagnetic wave propagate in seawater.

Then, the channel response of the seabed-rock-layer communication channel is analyzed by the case where the transceiver is in contact with the seabed $(h=0 \mathrm{~m})$. Figure 9 shows the simulation results of amplitude-frequency response in the case of communication range of $10 \mathrm{~m}, 40 \mathrm{~m}$, 


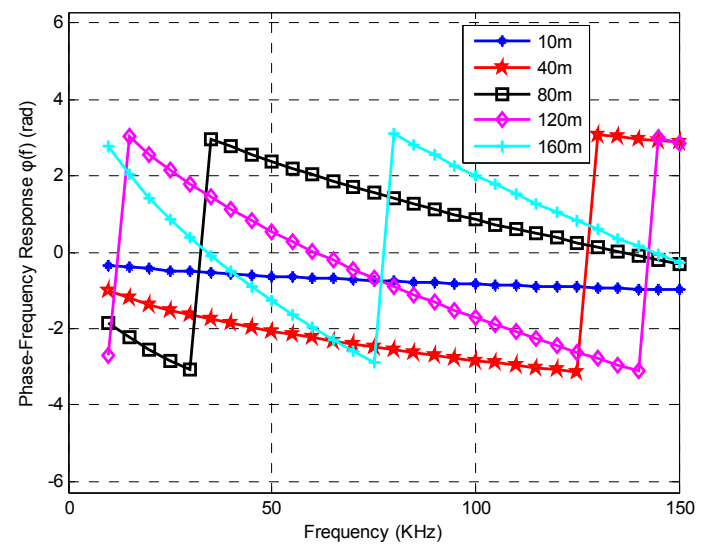

Fig. 10. Phase-frequency response.

$80 \mathrm{~m}, 120 \mathrm{~m}$ and $160 \mathrm{~m}$ in the operating frequency range of $10 \sim 150 \mathrm{kHz}$. It can be observed that the amplitude-frequency response of the channel exhibits a similar exponential trend, and the signal fading at different frequencies is different, the fading of the high-frequency signal is more serious than the low-frequency signal. In the case of communication range of $10 \mathrm{~m}$, the characteristic of the channel fading is relatively flat, and the maximum fading difference is only about 1.4 times. When the communication range is expanded to $80 \mathrm{~m}$, the fading difference is only about 14 times.

Figure 10 shows the simulation results of the phasefrequency response in the case of communication range of $10 \mathrm{~m}, 40 \mathrm{~m}, 80 \mathrm{~m}, 120 \mathrm{~m}$ and $160 \mathrm{~m}$ in the operating frequency range of $10 \sim 150 \mathrm{kHz}$. As can be seen from the figure, with the increase of the communication range in the simulated operating frequency range, the phase-frequency response curve is steeper, so that the speed of phase change is accelerated. For example, in the case of communication range of $10 \mathrm{~m}$, the phase difference between the $80 \sim 120 \mathrm{kHz}$ is only $0.139 \mathrm{rad}$, and in the case of the communication range of $120 \mathrm{~m}$, the difference is $1.545 \mathrm{rad}$. In the case of the range of $160 \mathrm{~m}$, the difference is close to $2.053 \mathrm{rad}$. According to the simulation results, we can see that the amplitude difference and phase difference are not serious in the short range, so that the transmission signal will not be severely affected.

\section{Conclusion}

In this paper, our research starts the analysis about the propagation characteristics of electromagnetic wave in seawater, we exploit the evanescent wave effect generated by total reflection to deduce and analyze the lateral $\mathrm{GH}$ Shift of electromagnetic wave on the seabed-rock-layer surface whose main component is basalt theoretically. A low loss seabed-rock-layer communication channel model for high speed communications has been established in this paper, whose propagation velocity can be increased 20 times and path loss can also be reduced to $1 / 20$ of the seawater condition. If we choose the appropriately lower frequency, communication range can be extended to sev- eral hundred meters or even several kilometers. This model is a breakthrough in the problem of limited communication range when electromagnetic wave propagates in seawater and also has certain significance for underwater shortrange and high speed reliable transmission.

\section{References}

[1] RHODES, M. Electromagnetic propagation in sea water and its value in military systems. In SEAS DTC Technical Conference. 2007, p. 1-6

[2] SOZER, E. M., STOJANOVIC, M., PROAKIS, J. G. Underwater acoustic networks. IEEE Journal of Oceanic Engineering, 2000, vol. 25 , no. 1 , p. $72-83$. DOI: $10.1109 / 48.820738$

[3] CHitre, M., ShahabUdEen, S., StOJANOVIC, M. Underwater acoustic communications and networking: Recent advances and future challenges. Marine Technology Society Journal, 2008, vol. 42, no. 1, p. 103-116. DOI: $10.4031 / 002533208786861263$

[4] COX JR, W. C. Simulation, Modeling, and Design of Underwater Optical Communication Systems. North Carolina State University, 2012. Available at: http://www.lib.ncsu.edu/resolver/1840.16/7588

[5] U. S. NAVY. Extremely low frequency transmitter site clam lake, Wisconsin. U. S. Navy Facts File, 2001. Available at: https://fas.org/nuke/guide/usa/c3i/fs_clam_lake_elf2003.pdf

[6] SHAW, A., Al-SHAMMA'A, A.I., WYLIE, S. R., et al. Experimental investigations of electromagnetic wave propagation in seawater. In European Microwave Conference. Manchester (UK), 2006, p. 572-575. DOI: 10.1109/EUMC.2006.281456

[7] AI-SHAMMA'A, A.I., SHAW, A., SAMAN, S. Propagation of electromagnetic waves at $\mathrm{MHz}$ frequencies through seawater. IEEE Transactions on Antennas and Propagation, 2004, vol. 52, no. 11 , p. 2843-2849. DOI: 10.1109/TAP.2004.834449

[8] ABDOU, A. A., SHAW, A., MASON, A., et al. Electromagnetic (EM) wave propagation for the development of an underwater Wireless Sensor Network(WSM). IEEE Sensors, 2011, vol. 25, no. 35 , p. 1571-1574. DOI: 10.1109/ICSENS.2011.6127319

[9] BUTLER, L. Underwater Radio Communication. Available at: http://users.tpg.com.au/users/ldbutler/Underwater_Communication .pdf

[10] GURU, B. S., HIZIROGLU, H. R. Electromagnetic Field Theory Fundamentals. $2^{\text {nd }}$ ed., Cambridge University Press, 2004. ISBN: 9781139165297

[11] CHAKRABORty, U., TEWARy, T., CHATTERJEe, R. P. Exploiting the loss-frequency relationship using RF communication in underwater communication networks. In International Conference on Computers and Devices for Communication. Kolkata (India), 2009, p.1-4.

[12] LI, J., TOUlgOAT, M., DEZIEL, M., et al. Propagation modeling and MAC-layer performance in EM-based underwater sensor networks. In Proceedings of the 4th ACM International Symposium on Development and Analysis of Intelligent Vehicular Networks and Applications. Montreal (Canada), 2014, p. 111-117. DOI: $10.1145 / 2656346.2656359$

[13] HUNT, K. P., NIEMEIER, J. J., KRUGER, A. RF communications in underwater wireless sensor networks. In IEEE International Conference on Electro/information Technology. Normal (IL, USA), 2010, p. 1-6. DOI: 10.1109/EIT.2010.5612087

[14] KELlEY, B., MANOJ, K., JAMSHIDI, M. Broadband RF communications in underwater environments using multi-carrier modulation. In Proceedings of the 2009 IEEE International 
Conference on Systems, Man and Cybernetics. San Antonio (USA), 2009, p. 2303-2308. DOI: 10.1109/ICSMC.2009.5345980

[15] KELLEY, B., NAISHADHAM, K. RF multicarrier signaling and antenna systems for low SNR broadband underwater communications. In IEEE Topical Conference on Biomedical Wireless Technologies, Networks, and Sensing Systems (BioWireleSS). Austin (USA), 2013, p. 240-242. DOI: 10.1109/BioWireleSS.2013.6613709

[16] MICHALSKI, K. A. Electromagnetic wave theory. Proceedings of the IEEE, 1987, vol. 75, no. 6, p. 862-863. DOI: 10.1109/PROC.1987.13818

[17] GOOS, F., HANCHEN, H. Ein neuer und fundamentaler Versuch zur Totalreflexion. Annalen Der Physik, 2010, vol. 436, no. 7, p. 333-346. DOI: $10.1002 /$ andp.19474360704

[18] SNYDER, A. W., LOVE, J. D. Goos-Hanchen shift. Applied Optics, 1976, vol. 15, no. 1, p. 236-238. DOI: 10.1364/AO.15.000236

[19] RESCH, K. J., LUNDEEN, J. S., STEINBERG, A. M. Total reflection cannot occur with a negative delay time. IEEE Journal of Quantum Electronics, 2001, vol. 37, no. 6, p. 794-799. DOI: $10.1109 / 3.922777$

[20] LIU, X. B., CAO, Z. Q., ZHU, P. F., et al. Simultaneously large and opposite lateral beam shifts for TE and TM modes on a double metal-cladding slab. Chinese Physics Letters, 2006, vol. 23, p. 2077-2079. DOI: $10.1088 / 0256-307 X / 23 / 8 / 032$

[21] STUBER, G. L. Principles of Mobile Communication. Kluwer Academic Publishers, 1996. ISBN: 978-1-4757-6268-6

[22] WANG, W. C. Electromagnetic Wave Theory. 1986. Available at: http://depts.washington.edu/mictech/optics/me557/week2.pdf.

[23] SHI, J., ZHANG S., YANG, C. J. High frequency RF based noncontact underwater communication. In IEEE Oceans. Yeosu (South Korea), 2012, p. 1-6. DOI: 10.1109/OCEANSYeosu.2012.6263403

\section{About the Authors ...}

Zhiqiang NIE was born in 1993. He received his B.S. degree in Communication Engineering from Hunan University in 2015 and currently is a master student at the Dept. of Electronic Science, National University of Defense Technology, China. His research interest is underwater electromagnetic communication.

Shilian WANG (corresponding author) was born in 1976. He received his $\mathrm{Ph} . \mathrm{D}$. degree in Information and Communication Engineering from the National University of Defense Technology, China in 2004. Currently he is a professor at the Dept. of Electronic Science, National University of Defense Technology. His primary interest is in the area of underwater communication and network confrontation.

Dahai CHEN has received the B.E. degree, M.E. degree and D.E. degree in Electronic Engineering from the University of Electronic Science and Technology in Chengdu (UESTC), Chengdu, China, in 1993, 2000, 2008, respectively. He joined the Institute of Electronic Engineering, China Academy of Engineering Physics since July, 1993. His main research interests are in the area of high data rate transmission systems design and implementation.

Tian DENG was born in 1993. She received her B.S. degree in Communication Engineering from Hunan University in 2015 and currently is a master student at the Dept. of Electronic Science, National University of Defense Technology, China. Her research interest is modern communication technology. 